Discussion Paper No. 535

\title{
STATIONARITY AND THE EXISTENCE OF MOMENTS OF A FAMILY OF GARCH PROCESSES*
}

\author{
Shiqing Ling \\ and \\ Michael McAleer
}

\author{
April 2001 \\ The Institute of Social and Economic Research \\ Osaka University \\ 6-1 Mihogaoka, Ibaraki, Osaka 567-0047, Japan \\ * To appear in Journal of Econometrics.
}




\title{
Stationarity and the Existence of $[$ Moments of a Family of GARCHProcesses *
}

\author{
Shiqing Ling \\ Department of Mathematics \\ Hong Kong University of Science and Technology \\ Michael McAleer \\ Department of Economics \\ University of Western Australia
}

Revised: February 2001

\begin{abstract}
This paper investigates some structural properties of a family of GARCH processes. A simple sufficient condition for the existence of the $\alpha \delta$-order stationary solution of the processes is derived, where $\alpha \in(0,1]$ and $\delta>0$. The solution is strictly stationary and ergodic, and the causal expansion of the family of GARCH processes is also established. Furthermore, the necessary and sufficient condition for the existence of the moments is obtained. The technique used in this paper for the moment conditions is different to that used in He and Terasvirta (1999a), and avoids the assumption that the process started at some finite value infinitely many periods ago. Moreover, the conditions for the strict stationarity of the model and the existence of its moments are simple to check and should prove useful in practice.
\end{abstract}

JEL classification: C22, C51

Keywords: Ergodicity; Existence of moments; GARCH; Stationarity.

*The authors would like thank Dick van Dijk and two referees for their helpful comments. The first author wishes to acknowledge the financial support of the Australian Research Council. The second author wishes to acknowledge the financial support of the Australian Research Council and the Institute of Social and Economic Research at Osaka University. 


\section{Introduction}

He and Terasvirta (1999a) defined the following general class of GARCH(1, 1) model:

$$
\varepsilon_{t}=z_{t} h_{t}, h_{t}^{\delta}=g\left(z_{t-1}\right)+c\left(z_{t-1}\right) h_{t-1}^{\delta}
$$

where $\operatorname{Pr}\left\{h_{t}^{\delta}>0\right\}=1, \delta>0, z_{t}$ is a sequence of i.i.d. random variables with mean

zero and variance 1 , and $g(x)$ and $z(x)$ are nonegative functions. As indicated by these authors, this family of GARCH processes includes the $\operatorname{GARCH}(1,1)$ model of Engle (1982) and Bollerslev(1986), the absolute value GARCH $(1,1)$ model of Taylor (1986) and Schwert (1989), the asymmetric GJR-GARCH $(1,1)$ model of Glosten et al. (1993) (which seems to be the most widely used specification for asymmetric time-varying volatility), the nonlinear $\operatorname{GARCH}(1,1)$ model of Engle (1990), the volatility switching GARCH $(1,1)$ model of Fornari and Mele (1997), the threshold GARCH $(1,1)$ model of Zakoian (1994), the fourth-order nonlinear generalized moving-average conditional heteroskedasticity (4NLGMACH(1,1)) model of Yang and Bewley (1995), and the generalized quadratic ARCH $(1,1)$ model of Sentana (1995).

It was argued by He and Terasvirta (1999a) that the necessary and sufficient condition for the $m \delta$ th unconditional moment of model (1.1) to exist is

$$
E\left[c\left(z_{t}\right)\right]^{m}<1
$$

where $m$ is a positive integer and $\delta=1$ or 2 . As in Engle (1982) and much of the subsequent literature, He and Terasvirta's (1999a) result in their Theorem 1 was derived under the following assumption:

Model (1.1) started at some finite value infinitely many periods ago.

Even though He and Terasvirta (1999a) restrict attention to the two integers $\delta=1,2$, their proofs actually hold for any $\delta>0$. Furthermore, He and Terasvirta (1999b) derived the fourth moment condition for the higher-order GARCH (p, q) model of 
Bollerslev (1996) (see also Karanasos (1999) for a related result). Ling and McAleer (1999) established the necessity and sufficiency of the condition for the existence of the fourth and higher moments for the GARCH (p, q) model.

The results in He and Terasvirta (1999a, b) are useful. However, two problems in their family of $\operatorname{GARCH}(1,1)$ processes have not yet been resolved. First, the condition under which model (1.1) is strictly stationary has not been established. Second, assumption (1.3) is impossible to check in practice, so that it is an axiom rather than an assumption. The purpose of this paper is to resolve these two problems. Without using assumption (1.3), we provide a simple sufficient condition for the strict stationarity of model (1.1), and establish the necessary and sufficient condition for the existence of its moments. These conditions are simple to check and should prove useful in practice.

\section{Main Results}

Theorem 2.1. If $E\left|z_{t}\right|^{\alpha \delta}<\infty, E\left[g\left(z_{t}\right)\right]^{\alpha}<\infty$ and $E\left[c\left(z_{t}\right)\right]^{\alpha}<1$ for some $\alpha \in$ $(0,1]$, then there exists a unique $\alpha \delta$-order stationary solution to (1.1). The solution is strictly stationary and ergodic, and has the following causal expansion:

$$
\varepsilon_{t}=z_{t} h_{t}, h_{t}^{\delta}=g\left(z_{t-1}\right)+\sum_{k=0}^{\infty} \prod_{j=0}^{k} c\left(z_{t-1-j}\right) g\left(z_{t-1-k}\right)
$$

where the infinite sum converges almost surely (a.s.).

Remark. Theorem 2.1 makes clear that the model given in (1.1) did, in fact, start infinitely many periods ago. However, this is a consequence of the existence of the unique stationary solution and is not an assumption.

When $g\left(z_{t-1}\right) \equiv \alpha_{0}>0$ and $c\left(z_{t-1}\right)=\alpha z_{t-1}^{2}+\beta$, namely Bollerslev's $\operatorname{GARCH}(1,1)$ model, the condition $E\left[c\left(z_{t}\right)\right]^{\alpha}<1$ for some $\alpha \in(0,1]$ is equivalent to $\ln \left[c\left(z_{t}\right)\right]<0$, which is the necessary and sufficient condition given in Nelson (1990) and Bougerol and Picard (1992).

Theorem 2.2. If $E\left|z_{t}\right|^{m \delta}<\infty$ and $E\left[g\left(z_{t}\right)\right]^{m \delta}<\infty$, then the necessary and 
sufficient condition for the existence of the moth moment of the solution $\left\{\varepsilon_{t}\right\}$ in Theorem 2.1 is

$$
E\left[c\left(z_{t}\right)\right]^{m \delta}<1
$$

where $m$ is a positive integer.

Note that, without strict stationarity, $E h_{t}^{k m}=E h_{t-n}^{k m}$ is used in (A.3) in He and Terasvirta (1999a), which means that the authors proved the necessity of the condition but not sufficiency. He and Terasvirta's (1999a) proof of necessity uses assumption (1.3), whereas the proof of necessity in this paper does not require this assumption. The proof of sufficiency in this paper uses the concept of Markov chains and Tweedie's (1988) criterion.

In general, the results in Theorem 2.2 cannot be easily extended to higher orders of the family of GARCH processes given in (1.1). However, for some special cases, such as the GARCH (p,q) model in Bollerslev (1986), the sufficient condition for the existence of moments is established in Chen and An (1998) and Ling (1999) (see Ling and McAleer (1999) for the proof of necessity). Ling and McAleer (1999) also established the necessary and sufficient conditions for the existence of moments for an asymmetric power GARCH (p,q) model in Ding et al. (1993), a special case of which is the widely used asymmetric GARCH $(1,1)$ model of Glosten et al. (1993) (this special case of model (1.1) is considered in Example 2.1 below).

In the following, we give two examples of the second and fourth moment conditions.

Example 2.1. In model (1.1), let $\delta=2, g\left(z_{t-1}\right) \equiv \alpha_{0}$, and $c\left(z_{t-1}\right)=\beta+(\alpha+$ $\left.\omega I\left(z_{t-1}\right)\right) z_{t-1}^{2}$, with $I\left(z_{t-1}\right)=1$ if $z_{t-1}<0$, and $I\left(z_{t-1}\right)=0$ otherwise, which is the asymmetric GJR-GARCH(1,1) model of Glosten et al. (1993).

(i) For this model, $E\left[c\left(z_{t-1}\right)\right]=\beta+\alpha+\omega b$, where $b=E\left[I\left(z_{t-1}\right) z_{t-1}^{2}\right]$. When $z_{t}$ follows a symmetric distribution, $b=1 / 2$ by direct calculation. In this case, the second moment condition for the asymmetric GJR-GARCH $(1,1)$ model, which is 
simple to check, is given by

$$
\beta+\alpha+\frac{1}{2} \omega<1
$$

When $\omega=0$, condition (2.1) reduces to the well-known second moment condition for the $\operatorname{GARCH}(1,1)$ model. Condition (2.1) means that the admissible region of $(\alpha, \beta)$ for second-order stationarity of the asymmetric GJR-GARCH(1,1) model is smaller than that for the symmetric $\operatorname{GARCH}(1,1)$ model, as the asymmetry of the model increases its uncertainty.

(ii) Furthermore, if $z_{t} \sim N(0,1)$, the fourth moment condition for the asymmetric GJR-GARCH $(1,1)$ model is given by

$$
\beta^{2}+2 \beta \alpha+3 \alpha^{2}+\beta \omega+3 \alpha \omega+\frac{3}{2} \omega^{2}<1
$$

When $\omega=0$, condition (2.2) reduces to the well-known fourth moment condition for the $\operatorname{GARCH}(1,1)$ model.

(iii) If $z_{t} \sim t(\nu)$ with $\nu \geq 5$, the fourth moment condition is given by

$$
\beta^{2}+2 \beta \alpha+s \alpha^{2}+\beta \omega+\frac{s}{2}\left(2 \alpha \omega+\omega^{2}\right)<1
$$

where $s=3(\nu-2) /(\nu-4)$. When $\nu \rightarrow \infty$, condition $(2.3)$ reduces to condition $(2.2)$.

Example 2.2. In model (1.1), let $\delta=2, g\left(z_{t-1}\right) \equiv \alpha_{0}$, and $c\left(z_{t-1}\right)=\beta+$ $\alpha\left[1-2 \eta \operatorname{sgn}\left(z_{t-1}\right)+\eta^{2}\right] z_{t-1}^{2}$, namely the nonlinear $\operatorname{GARCH}(1,1)$ model of Engle (1990), which is a special case of the asymmetric power GARCH model of Ding et al. (1993).

(i) For this model, $E\left[c\left(z_{t-1}\right)\right]=\beta+\alpha+\alpha \eta^{2}-2 \alpha \eta b$, where $b=E\left[\operatorname{sgn}\left(z_{t-1}\right) z_{t-1}^{2}\right]$. When $z_{t}$ follows a symmetric distribution, $b=0$ and, in this case, the second moment condition for the nonlinear $\operatorname{GARCH}(1,1)$ model is given by

$$
\beta+\alpha+\alpha \eta^{2}<1
$$

When $\eta=0$, condition (2.4) reduces to that for $\operatorname{GARCH}(1,1)$. 
(ii) Furthermore, if $z_{t} \sim N(0,1)$, the fourth moment condition for the nonlinear $\operatorname{GARCH}(1,1)$ model is given by

$$
\beta^{2}+2 \beta \alpha\left(1+\eta^{2}\right)+3 \alpha^{2}\left[\left(1+\eta^{2}\right)^{2}+4 \eta^{2}\right]<1
$$

When $\eta=0$, condition (2.5) reduces to the fourth moment condition for $\operatorname{GARCH}(1,1)$.

(iii) If $z_{t} \sim t(\nu)$ with $\nu \geq 5$, the fourth moment condition is given by

$$
\beta^{2}+2 \beta \alpha\left(1+\eta^{2}\right)+s \alpha^{2}\left[\left(1+\eta^{2}\right)^{2}+4 \eta^{2}\right]<1
$$

where $s=3 \nu^{2} /[(\nu-4)(\nu-2)]$. When $\nu \rightarrow \infty$, condition $(2.6)$ reduces to condition (2.5). The comments in Example 2.1 relating to the reason for the increased uncertainty arising from asymmetry remain valid for the nonlinear $\operatorname{GARCH}(1,1)$ model.

\section{Proofs of the Main Results}

Proof of Theorem 2.1. Let $S_{n}=g\left(z_{t-1}\right)+\sum_{k=0}^{n} \prod_{j=0}^{k} c\left(z_{t-1-j}\right) g\left(z_{t-1-k}\right)$. Then

$$
\begin{aligned}
E\left(\lim _{n \rightarrow \infty} S_{n}\right)^{\alpha} & \leq E\left\{\left[g\left(z_{t-1}\right)\right]^{\alpha}+\sum_{k=1}^{\infty}\left[\prod_{j=1}^{k} c\left(z_{t-1-j}\right) g\left(z_{t-1-k}\right)\right]^{\alpha}\right\} \\
& =E\left[g\left(z_{t-1}\right)\right]^{\alpha}+\sum_{k=1}^{\infty} E\left[\prod_{j=1}^{k} c\left(z_{t-1-j}\right)\right]^{\alpha} E\left[g\left(z_{t-1-k}\right)\right]^{\alpha} \\
& =E\left[g\left(z_{t-1}\right)\right]^{\alpha}\left[1+\sum_{k=0}^{\infty}\left\{E\left[c\left(z_{t-1}\right)\right]^{\alpha}\right\}^{k+1}\right] \\
& =E\left[g\left(z_{t-1}\right)\right]^{\alpha}\left[1-E\left[c\left(z_{t-1}\right)\right]^{\alpha}\right]^{-1}<\infty,
\end{aligned}
$$

so that $S_{n}$ converges a.s. under the given assumptions. Denote the limit of $S_{n}$ by $h_{t}^{\delta}$, that is,

$$
h_{t}=\left[g\left(z_{t-1}\right)+\sum_{k=0}^{\infty} \prod_{j=0}^{k} c\left(z_{t-1-j}\right) g\left(z_{t-1-k}\right)\right]^{1 / \delta} .
$$

It is easy to verify that $h_{t}$ is a solution of (1.1) and $E h_{t}^{\alpha \delta}<\infty$. From (3.1), $h_{t}$ is a fixed functional of a sequence of i.i.d. random variables, and hence is also strictly stationary and ergodic, in which case so is $\varepsilon_{t}$. 
To prove uniqueness, let $h_{1 t}$ be another $\alpha \delta$ th-order stationary solution of model (1.1). Then

$$
\begin{aligned}
E\left|h_{t}^{\delta}-h_{1 t}^{\delta}\right|^{\alpha} & =E\left[c\left(z_{t-1}\right)\right]^{\alpha} E\left|h_{t-1}^{\delta}-h_{1 t-1}^{\delta}\right|^{\alpha} \\
& \leq\left\{E\left[c\left(z_{t-1}\right)\right]^{\alpha}\right\}^{k}\left(E h_{t-k}^{\alpha \delta}+E h_{1 t-k}^{\alpha \delta}\right) \rightarrow 0,
\end{aligned}
$$

as $k \rightarrow \infty$. Thus, $h_{t}=h_{1 t}$ a.s.. This completes the proof.

Proof of the necessity of Theorem 2.2. It is easy to show that

$$
\begin{aligned}
E h_{t}^{\delta m} & \geq E\left[g\left(z_{t-1}\right)\right]^{m}+E\left[c\left(z_{t-1}\right)\right]^{m} E h_{t-1}^{\delta m} \\
& \geq E\left[g\left(z_{t-1}\right)\right]^{m}\left[1+\sum_{i=0}^{k}\left\{E\left[c\left(z_{t-1}\right)\right]^{m}\right\}^{i}\right] .
\end{aligned}
$$

Since $E h_{t}^{\delta m}<\infty$, the above inequality holds only if $E\left[g\left(z_{t-1}\right)\right]^{m}<\infty$ and $E\left[c\left(z_{t-1}\right)\right.$ ]$^{m}<1$. This completes the proof.

The proof of the sufficiency of Theorem 2.2 needs the concept of Markov chains and Tweedie's (1988) criterion. Let $\left\{X_{t} ; t=1,2, \cdots\right\}$ be a temporally homogeneous Markov chain with a locally compact completely separable metric state space $(S, \mathcal{B})$. The transition probability is $P(x, A)=\operatorname{Pr}\left(X_{n} \in A \mid X_{n-1}=x\right)$, where $x \in S$ and $A \in \mathcal{B}$. Tweedie's criterion is given in the following lemma.

Lemma 3.1. (Tweedie, 1988, Theorem 2) Suppose that $\left\{X_{t}\right\}$ is a Feller chain. (1) If there exists, for some compact set $A \in \mathcal{B}$, a non-negative function $f$ and $\varepsilon>0$ satisfying

$$
\int_{A^{c}} P(x, d y) f(y) \leq f(x)-\varepsilon, \quad x \in A^{c},
$$

then there exists a $\sigma$-finite invariant measure $\mu$ for $P$ with $0<\mu(A)<\infty$; (2)Furthermore, if

$$
\int_{A} \mu(d x)\left[\int_{A^{c}} P(x, d y) f(y)\right]<\infty
$$

then $\mu$ is finite, and hence $\pi=\mu / \mu(S)$ is an invariant probability; (3) Furthermore, if

$$
\int_{A^{c}} P(x, d y) f(y) \leq f(x)-g(x), \quad x \in A^{c},
$$


then $\mu$ admits a finite $g$-moment, that is,

$$
\int_{S} \mu(d y) g(y)<\infty
$$

It is clear that any solution $h_{t}^{\delta}$ of model (1.1) is a Markov chain with state space $R_{0}=(0, \infty)$. Tweedie's criterion can be used to find the sufficient condition for the existence of a stationary and finite $g$-moment solution of model (1.1). Now we give a lemma that is used in the proof of the sufficiency of Theorem 2.2.

Lemma 3.2. There exists an interval $A=(0, K)$ such that the function $f$ defined by

$$
f(x)=1+x^{m}
$$

satisfies

$$
E\left[f\left(h_{t}^{\delta}\right) \mid h_{t-1}^{\delta}=x\right] \leq \Delta, \quad x \in A
$$

and

$$
E\left[f\left(h_{t}^{\delta}\right) \mid h_{t-1}^{\delta}=x\right] \leq(1-\tau) f(x), x \in A^{c},
$$

where $A^{c}=[K, \infty), \Delta$ is a positive constant, and $\tau$ and $K$ are constants not depending on $x$.

Proof. From the given conditions, we can show that

$$
\begin{aligned}
E\left[f\left(h_{t}^{\delta}\right) \mid h_{t-1}^{\delta}=x\right] & =1+E\left[g\left(z_{t-1}\right)+c\left(z_{t-1}\right) x\right]^{m} \\
& \leq 1+E\left[c\left(z_{t-1}\right)\right]^{m} x^{m}+b(1+x)^{m-1} \\
& \leq f(x)\left[1-\frac{\tau_{1} x^{m}+b(1+x)^{m-1}}{1+x^{m}}\right]
\end{aligned}
$$

where $\tau_{1}=1-E\left[c\left(z_{t-1}\right)\right]^{m}$, and $b=\sum_{i=0}^{m-1}\left(\begin{array}{c}m \\ i\end{array}\right) E^{m-i}\left[g\left(z_{t-1}\right)\right] E^{i}\left[c\left(z_{t-1}\right)\right]$.

Note that $\tau_{1} \in(0,1)$, and $x^{m} /\left(1+x^{m}\right)$ and $b(1+x)^{m-1} /\left(1+x^{m}\right)$ converge to 1 and 0 , respectively, as $x \rightarrow \infty$. It is obvious that there exists a constant $K$ such that, when $x \geq K,\left[\tau_{1} x^{m}+b(1+x)^{m-1}\right] /\left(1+x^{m}\right)>\tau_{1} / 2-\tau_{1} / 4>0$. Denote $A=(0, K)$. Let $\tau=\tau_{1} / 4$ and $\Delta=1+K^{m}$. It follows that

$$
E\left[f\left(h_{t}^{\delta}\right) \mid h_{t-1}^{\delta}=x\right] \leq \Delta, \quad x \in A
$$


and

$$
E\left[f\left(h_{t}^{\delta}\right) \mid h_{t-1}^{\delta}=x\right] \leq(1-\tau) f(x), x \in A^{c}
$$

This completes the proof.

Proof of the sufficiency of Theorem 2.2. First, note that $\left\{h_{t}^{\delta}\right\}$ is a Markov chain with state space $R_{0}$. It is straightforward to prove that, for each bounded continuous function $f$ on $R_{0}, E\left[f\left(h_{t}^{\delta}\right) \mid h_{t-1}^{\delta}=x\right]$ is continuous in $x$, that is, $\left\{h_{t}^{\delta}\right\}$ is a Feller chain.

From Lemma 3.2, there exists a constant $K>0, A=(0, K)$ and $\tau>0$ such that the function defined by $f(x)=1+x^{m}$ satisfies

$$
E\left[f\left(h_{t}^{\delta}\right) \mid h_{t-1}^{\delta}=x\right] \leq \Delta, x \in A
$$

and

$$
E\left[f\left(h_{t}^{\delta}\right) \mid h_{t-1}^{\delta}=x\right] \leq(1-\tau) f(x), x \in A^{c},
$$

where $\tau$ and $\Delta$ are positive constants.

By (3.7)-(3.8), it is not difficult to show that the function $f(x)$ satisfies conditions (3.2) and (3.3) in Lemma 3.1 and, hence, there is an invariant probability measure $\pi$ for the process $\left\{h_{t}^{\delta}\right\}$.

Let $g(x)=\tau f(x)$. By (3.8) and Lemma $3.1(3)$, we know that $E_{\pi} f\left(h_{t}^{\delta}\right)$ is finite. Thus, $E_{\pi} h_{t}^{\delta m}<\infty$.

By Hölder's inequality, $E_{\pi} h_{t}^{\alpha \delta} \leq\left(E_{\pi} h_{t}^{\delta m}\right)^{\alpha / m}<\infty$, and hence $\pi$ is the $\alpha \delta$ th-order stationary distribution of $h_{t}$. Thus, $\varepsilon_{t}=z_{t} h_{t}$ is the $\alpha \delta$ th-order stationary solution of model (1.1). By Theorem 2.1, the solution is unique. Thereforce, the solution $\left\{\varepsilon_{t}\right\}$ in Theorem 2.1 has finite $m$ th-order moment. This completes the proof. 


\section{REFERENCES}

Bollerslev, T. (1986) Generalized autoregressive conditional heteroskedasticity. Journal of Econometrics 31, 307-327.

Bougerol, P. and Picard, N.M. (1992) Stationarity of GARCH processes and of some nonnegative time series. Journal of Econometrics 52, 115-127.

Chen, M. amd An, H.Z. (1998) A note on the stationarity and the existence of moments of the GARCH model. Statistica Sinica 8, 505-510.

Ding, Z., Granger, C.W.J., and Engle, R.F. (1993) A long memory property of stock market returns and a new model. Journal of Empirical Finance 1, 83106.

Engle, R.F. (1982) Autoregressive conditional heteroskedasticity with estimates of the variance of United Kingdom inflation. Econometrica 50, 987-1007.

Engle, R. F. (1990) Discussion: stock market volatility and the crash of 87. Review of Financial Studies 3, 103-106.

Fornari, F. and Mele, A. (1997) Sign- and volatility-switching ARCH models: theory and applications to international stock markets. Journal of Applied Econometrics 12, 1779-1801.

Glosten, L.R., Jagannathan, R. and Runkle, D. (1993). On the relation between the expected value and the volatility of the nominal excess return on stocks. Journal of Finance 48, 1779-1801.

He, C. and Terasvirta, T. (1999a) Properties of moments of a family of GARCH processes. Journal of Econometrics 92, 173-192.

He, C. and Terasvirta, T. (1999b) Fourth moment structure of the GARCH (p, q) process. Econometric Theory, 15, 824-846. 
Karanasos, M. (1999) The second moment and the autocovariance function of the squared errors of the GARCH model. Journal of Econometrics 90, 63-76.

Ling, S. (1999) On the probabilistic properties of a double threshold ARMA conditional heteroskedasticity model. Journal of Applied Probability 36, 1-18.

Ling, S. and McAleer, M. (1999) Necessary and sufficient moment conditions for the GARCH (r, s) and Asymmetric Power GARCH (r, s) models. Submitted.

Nelson, D.B. (1990) Stationarity and persistence in the GARCH (1,1) model. Econometric Theory 6, 318-334.

Schwert, G. W. (1989) Why does stock market volatility change over time? Journal of Finance 45, 1129-1155.

Sentana, E. (1995) Quadratic ARCH models. Review of Economic Studies 62, 639-661.

Taylor, S. (1986) Modelling Financial Time Series. Wiley, New York.

Tweedie, R.L. (1988) Invariant measure for Markov chains with no irreducibility assumptions. Journal of Applied Probability 25A, 275-285.

Yang, M. and Bewley, R. (1995) Moving average conditional heteroskedastic processes. Economics Letters 49, 367-372.

Zakoian, J.M. (1994) Threshold heteroskedastic models. Journal of Economic Dynamics and Control 18, 931-955. 